\title{
FGF2 promotes metastasis of uveal melanoma cells via store-operated calcium entry
}

This article was published in the following Dove Press journal:

OncoTargets and Therapy

8 November 2017

Number of times this article has been viewed

Yanyan Wang'

Xiuli Bao ${ }^{2}$

Zhiyong Zhang ${ }^{3}$

Yi Sun ${ }^{4}$

Xiyuan Zhou'

'Department of Ophthalmology, The Second Affiliated Hospital of Chongqing Medical University, Chongqing, ${ }^{2}$ Department of Ophthalmology, ${ }^{3}$ Department of Nuclear Medicine, ${ }^{4}$ Department of Ophthalmology, Affiliated Hospital of Inner Mongolia Medical University, Hohhot, Inner Mongolia, People's Republic of China
Correspondence: Xiyuan Zhou Department of Ophthalmology, The Second Affiliated Hospital of Chongqing Medical University, Chongqing 400010 ,

People's Republic of China

Tel +86 I399628 6679

Email zhouxiyuan2002@aliyun.com

\begin{abstract}
Uveal melanoma (UM), the most common primary intraocular malignancy in adults, is highly metastatic and associated with dismal prognosis. Fibroblast growth factor 2 (FGF2) has been shown to induce cell proliferation and angiogenesis of melanoma and other malignancies. However, the expression of FGF2 in UM and its effects on melanoma cell migration are not well known. In this study, we found FGF2 expression was related to UM histological subtype and presence of metastasis. In vitro experiments showed that FGF2 treatment caused increased horizontal and vertical migration and F-actin cytoskeleton assembly as well as decreased adhesive activity of MUM2B cells, together with increased intracellular calcium concentration and expression of ORAI1 and STIM1 - two key regulatory proteins of store-operated calcium entry (SOCE). The mouse xenograft model showed that MUM2B cells with FGF2 stimulation grew into larger tumor masses and were prone to metastasis. In addition, the SOCE inhibitor 2-aminoethoxydiphenyl borate (2-APB) reversed all of these effects of FGF2. Finally, human UM samples and mouse xenograft model samples were used to confirm the correlation of FGF2 with ORAI1 and STIM1 expression. Taken together, our study suggests that FGF2 promotes metastasis of UM via SOCE.
\end{abstract}

Keywords: FGF2, uveal melanoma, metastasis, store-operated calcium entry, ORAI1, STIM1

\section{Introduction}

Uveal melanoma (UM) is the most common adult primary malignant intraocular tumor, and represents approximately $5 \%$ of all reported melanomas. ${ }^{1}$ Although various options are available for the treatment of UM, including radioactive plaque therapy, transpupillary thermotherapy and proton beam radiotherapy, the prognosis for patients is unfavorable, especially in the metastatic phase. ${ }^{2}$ Patients with UM who have hepatic or pulmonary metastasis have a median survival of only a few months, and treatment options at the disseminated stage are still very limited. ${ }^{3}$ Thus, it is an urgent necessity to understand the metastatic mechanisms of UM and to develop modalities that prevent dissemination of tumor cells if we are to improve survival of patients with UM.

The fibroblast growth factor 2 (FGF2) is one of the 23 members of the FGF family known to modulate a variety of biological processes, including survival, proliferation, motility, differentiation, and angiogenesis. ${ }^{4-6}$ Experimental and clinical studies highlight FGF2 overexpression in a variety of tumors, including breast, lung, and prostate cancer. ${ }^{7-9}$ FGF2-overexpressing melanoma cells exhibit marked proliferation, upward migration, cluster formation, and type IV collagen expression within the epidermal compartment. ${ }^{10-12}$ Interferences with the FGF2/FGFR pathway resulted in impaired neovascularization and growth of human melanoma xenografts, ${ }^{13}$ demonstrating that FGF2 is essential in melanoma progression and may be an interesting target to explore 
for antitumor approaches. While the expression of FGF2 has been associated with UM cell proliferation, the other roles of FGF2 are widely unexplored in UM so far.

Activated FGF2/FGFR signaling enables the binding site of phospholipase $\mathrm{C} \gamma$ (PLC $\gamma$ ) to recruit and activate PLC $\gamma$ for the catalysis of phosphatidylinositol diphosphate (PIP2) to diacylglycerol (DAG) and inositol triphosphate (IP3). ${ }^{14}$ In general, activation of IP3 evokes $\mathrm{Ca}^{2+}$ release from the endoplasmic reticulum (ER) store. The resulting decrease of $\mathrm{Ca}^{2+}$ concentration in the ER is sensed by the stromal interaction molecules (STIM), which then translocate to the plasma membrane, where they interact with ORAI $\mathrm{Ca}^{2+}$ channel subunits, leading to $\mathrm{Ca}^{2+}$ influx. ${ }^{15}$ This process is referred to as store-operated $\mathrm{Ca}^{2+}$ entry (SOCE). SOCE, within normal and cancer cells, has been increasingly implicated in many important cellular functions such as migration, proliferation, differentiation, and cytokine secretion and, yet, the underlying mechanisms remain largely unknown. ${ }^{16,17}$ Qi et al has reported that FGF4 could induce epithelial-mesenchymal transition by inducing SOCE in lung adenocarcinoma cells. ${ }^{18}$ However, as the most extensively examined role in the FGF family, FGF2 in regulating SOCE remains to be answered.

In this study, the expression pattern and clinicopathologic significance of FGF2 were analyzed on an array of 32 human UM cases. We studied the effects of FGF2, in a UM cell line MUM2B, on horizontal and vertical migration, adhesion abilities, and F-actin cytoskeleton assembly in vitro as well as on metastatic ability in an animal xenograft model. In addition, we treated FGF2-stimulated cells with 2-APB, a SOCE inhibitor, and determined the migration ability and expression of SOCE-regulatory proteins (ORAI1 and STIM1) to further verify the SOCE-inducing effect of FGF2 in UM cells.

\section{Materials and methods}

\section{Tissue samples}

In this study, we analyzed tumors from 32 patients with UM who had been treated by primary enucleation without prior radiation at the Ophthalmology Department of the Second Affiliated Hospital of Chongqing Medical University (Chongqing, People's Republic of China) between January 2005 and December 2013. The use of the tissue samples in this study was approved by the Institutional Research Committee. Clinicopathologic data including age, gender, largest tumor diameter (LTD), height, histological subtype, and occurrence of metastasis were obtained from the clinical records and pathological reports of patients. The 32 patients with UM had a median age of 61 years (range 42-75) at the time of surgical treatment. The tumors had a mean LTD of $13.4 \mathrm{~mm}$ (range 9.5-20.2 $\mathrm{mm}$ ) and a mean height of $8.5 \mathrm{~mm}$ (range 2.6-14.1 mm). Thirty cases showed scleral invasion and five showed extrascleral extension; 14 cases showed ciliary body involvement; PAS (periodic acid-schiff stain)positive loops were detected in 11 cases. The ciliary body was involved in 14 cases, and vasculogenic mimicry was detected in 16 cases. Follow-up time ranged from 12 to 80 months with a median of 36.6 months.

\section{Hematoxylin-eosin (H\&E) staining and immunohistochemical staining}

The melanin pigments can hinder antibody-antigen interactions in immunohistochemistry and obscure cell morphology. ${ }^{19}$ Heavily pigmented melanoma samples were treated with $3 \%$ warm hydrogen peroxide $\left(\mathrm{H}_{2} \mathrm{O}_{2}\right)$ at $55^{\circ} \mathrm{C}$ for $2 \mathrm{~h}$ to achieve the optimal and complete melanin-bleaching condition. Tissues were fixed in 10\% neutral-buffered formalin for $24 \mathrm{~h}$, embedded in paraffin wax, and cut into $4 \mu \mathrm{m}$ thicknesses. The paraffin-embedded sections were deparaffinized in xylene, and processed with a graded ethanol series. For H\&E staining, sections were stained with hematoxylin and eosin sequentially. For immunohistochemical staining, the sections were pretreated with microwaves in citrate phosphate buffer, blocked with $0.3 \% \mathrm{H}_{2} \mathrm{O}_{2}$, and incubated with a series of antibodies (FGF2) 1:100; STIM1 1:100; ORAI1 1:50 (all three antibodies from Santa Cruz Biotechnology, CA, USA) overnight at $4^{\circ} \mathrm{C}$. Then, sections immunostained with HRP-conjugated antibody (Zhongshan Chemical Co., Beijing, People's Republic of China) for $40 \mathrm{~min}$ at $37^{\circ} \mathrm{C}$ were revealed using 3,3-diaminobenzidine buffer as substrate. PBS was used as the negative control. The expression of FGF2, STIM1, and ORAI1 was analyzed, and considered immunoreactive when brown granules were identified in the cytoplasm of UM tumor cells. For each specimen, expressions of FGF2, STIM1, and ORAI1 were quantified using a visual grading system based on the extent of staining and the intensity of staining. Staining intensity was determined as 0 , negative; 1 , weak; 2 , moderate; and 3, strong. The percentage of positive cells was defined as 0 , negative; $1,1 \%-10 \%$ positive cells; $2,11 \%-50 \%$ positive cells; $3,51 \%-80 \%$ positive cells; and $4,>80 \%$ positive cells. An immunoreactive score value $\geq 4$ was considered a positive staining result.

\section{Cell culture}

MUM2B is a human UM cell line isolated from liver metastasis and selected for its highly aggressive phenotype. ${ }^{20}$ MUM2B cell lines were negative for BRAF V599E mutation. ${ }^{20}$ 
MUM2B cells are highly invasive in membrane invasion culture system assays, can deform floating collagen gels, and can form vasculogenic mimicry patterns in three-dimensional cultures. MUM2B cells could produce more IL-1 than the less aggressive primary cells. Moreover, it produces more IL-6 and TNF- $\alpha$ and other cytokines implicated in the progression of UM. ${ }^{21}$ The human UM cell line MUM2B was obtained from the China Infrastructure of Cell Line Resources (Beijing, People's Republic of China). Cells were cultured in Roswell Park Memorial Institute (RPMI)-1640 media supplemented with $10 \%$ fetal bovine serum (FBS; Hyclone, Logan, UT, USA) at $37^{\circ} \mathrm{C}$ in a $5 \% \mathrm{CO}_{2}$ incubator. In some in vitro experiments, after $24 \mathrm{~h}$ of starvation in serum-free medium, cells were stimulated with 2-APB $(20 \mu \mathrm{M}$; Abcam, Cambridge, $\mathrm{UK}$ ) for $12 \mathrm{~h}$ and then treated with FGF2 (10 ng/mL; R\&D Systems Inc., Minneapolis, MN, USA) for $24 \mathrm{~h}$.

\section{Intracellular calcium measurement}

Intracellular calcium concentration was measured by Fluo 3-AM as a calcium indicator using flow cytometry. Briefly, cells were washed with Hank's balanced salt solution (HBSS) and incubated with $1 \mathrm{~mL}$ HBSS containing $5 \mu \mathrm{mol} / \mathrm{L}$ Fluo 3-AM (Dojindo Laboratories, Kumamoto, Japan) at $37^{\circ} \mathrm{C}$ for $1 \mathrm{~h}$. Cells were washed and suspended in PBS. The intracellular calcium concentration in a population of 50,000 cells was measured by flow cytometry.

\section{Western blot analysis}

Protein $(50 \mu \mathrm{g} /$ lane $)$ was separated by $10 \%$ polyacrylamide gel electrophoresis and transferred onto polyvinylidene difluoride (PVDF) membranes. Blots were blocked and incubated with primary antibodies (STIM1 1:200, ORAI1 1:200, and $\beta$-actin 1:2,000) overnight at $4^{\circ} \mathrm{C}$. Subsequently, blots were washed and labeled with goat anti-rabbit IgG-HRP (Santa Cruz Biotechnology; 1:5,000) for $1 \mathrm{~h}$ at room temperature. Immunoreactive bands were visualized with ECL Western blot substrate. The relative density of bands was analyzed using an Odyssey infrared scanner (LI-COR Bioscience, Lincoln, NE, USA).

\section{Wound-healing assay}

Cells were plated in $35 \mathrm{~mm}$ dishes to form a monolayer 1 day before the assay. After making a uniform scratch with a $10 \mu \mathrm{L}$ pipette tip, the wells were washed with PBS. The dishes were incubated in a minimum medium (containing $0.5 \% \mathrm{FBS}$ ) at $37^{\circ} \mathrm{C}$ with $5 \% \mathrm{CO}_{2}$ for $24 \mathrm{~h}$. Cell motility was assessed by measuring the speed of wound closure at intervals. Images were taken at four time points: 0,12 , and $24 \mathrm{~h}$ after the scratch was applied.

\section{Boyden chamber assay}

Twenty four-well plates with uncoated inserts ( $8 \mu \mathrm{m}$ pore; BD Biosciences San Jose, CA, USA) were used to assess vertical motility of MUM2B cells. Briefly, $600 \mu \mathrm{L}$ culture medium supplemented with FGF2 was added to the lower part of the chamber; cells $\left(1 \times 10^{5}\right.$ cells $)$ in $200 \mu \mathrm{L}$ culture medium were seeded into the upper part. For the FGF2+2-APB group, the cells were pretreated by stimulation with 2-APB for $12 \mathrm{~h}$. After incubation at $37^{\circ} \mathrm{C}$ with $5 \% \mathrm{CO}_{2}$ for $24 \mathrm{~h}$, the passaged cells were fixed and visualized with crystal violet staining.

\section{Cell adhesion assay}

Sterile cover slips were coated overnight at $4^{\circ} \mathrm{C}$ with $1 \mathrm{~mL}$ of a solution containing $1 \mathrm{mg}$ fibronectin from human plasma (Sigma-Aldrich Co., St Louis, MO, USA) and put into $35 \mathrm{~mm}$ dishes. Then, $1 \mathrm{~mL}$ cell suspension $\left(4 \times 10^{5}\right.$ cells/ $\mathrm{mL}$ ) was added on the cover slips. Ice-cold PBS was added into the dishes to terminate the reaction after incubation for 5,15 , or $30 \mathrm{~min}$. Then, the cover slips were fixed in ice-cold $4 \%$ paraformaldehyde (PFA) and washed twice with PBS. After the nonbound cells were washed away, the bound cells were stained with crystal violet and semi-quantitated under a microscope $(\times 400)$.

\section{F-actin staining}

Cells were cultured on sterile glass cover slips and placed in serum-free medium 1 day prior to staining. Cells were fixed with $4 \%$ PFA for $10 \mathrm{~min}$ and quenched with $50 \mathrm{mM} \mathrm{NH}_{4} \mathrm{Cl}$ for $5 \mathrm{~min}$ and $0.2 \%$ Triton for $10 \mathrm{~min}$. The slips were incubated with the phalloidin conjugated to Alexa 594 (Sigma-Aldrich Co., Ltd.) for $40 \mathrm{~min}$ in the dark at room temperature, and then counterstained with diamidino-phenyl-indole for $10 \mathrm{~min}$. Cells were washed five times with PBS and visualized with confocal laser scanning microscopy (Leica TCS SP5; Leica Microsystems, Wetzlar, Germany).

\section{Mice and tumors}

All animal care and handling procedures were approved by the Institutional Animal Use and Care Committee of Chongqing Medical University. We administered $3 \times 10^{6}$ MUM2B cells in $100 \mu \mathrm{L}$ PBS via subcutaneous injection into the right groin of female nude mice (Wei Tong Li Hua Experimental Company, Beijing, People's Republic of China). In the FGF2 and FGF2/2-APB groups, mice were given subcutaneous injections of FGF2 (500 ng/mouse) three times per week. In the FGF2/2-APB group, mice were co-treated with 2 -APB $(20 \mathrm{mg} / \mathrm{kg})$ three times per week. Tumor volume was calculated using the equation volume $\left(\mathrm{mm}^{3}\right)=\left(\right.$ length $\times$ width $\left.^{2}\right) / 2$. All mice were euthanized on 
Day 30 post injection. Tumor masses were fixed, embedded, made into tissue sections, and then subjected to both H\&E and immunohistochemical staining.

\section{Statistical analysis}

GraphPad Prism was used for statistical calculations. Data are the result of at least triplicate independent determinations and presented as mean \pm SD. Differences between groups were assessed by two-tailed Student's $t$-test. Associations between FGF2 expression and clinicopathologic parameters and the differential expression of STIM1 and ORAI1 in FGF2-positive and -negative groups were assessed with Fisher's exact test and chi-square test. $P<0.05$ was considered statistically significant.

\section{Results}

\section{Correlation between FGF2 and} clinicopathologic characteristics of UM

The expression patterns of FGF2 were examined on an array of 32 human lung UM cases. Among these 32 samples, $18(56.3 \%)$ showed positive FGF2 expression (Figure 1A). Relationships between FGF2 expression and each clinicopathologic parameter are summarized in Table 1. FGF2 expression was strongly correlated with histological subtype $(P<0.05)$ and metastasis $(P<0.05)$. The frequency of positive FGF2 expression was higher in mixed/epithelioid samples than in spindle cell type samples. Twelve (37.5\%) patients with UM experienced metastasis. Patients with positive FGF2 expression had a higher rate of metastasis (10/12, 83.3\%) than those with negative FGF2 expression (2/14, 14.3\%). No correlations were found between FGF2 expression level and patient age or gender, LTD, or height. Moreover, we analyzed FGF2 expression in six sets of matched specimens (including UM primary foci and pulmonary or hepatic metastatic foci) obtained from one patient. Immunohistochemical staining of primary lesions and metastases revealed a heterogeneous distribution of FGF2, with elevated expression in tumor metastases (Figure 1B), further implying that FGF2 may be involved in the progression of UM.

\section{FGF2 elevates intracellular calcium concentration and increases the expression of STIMI and ORAII}

As the ubiquitous second messenger calcium regulates nearly every cellular event involved in tumorigenesis, we explored the potential role of FGF2 in the modulation of cellular $\mathrm{Ca}^{2+}$ homeostasis. The Fluo-3AM flow cytometry experiments demonstrated that the mean fluorescence intensity (MFI) of the cells with FGF2 treatment was significantly higher than the MFI of the control cells. ( $P<0.05$; Figure $2 \mathrm{~A})$.

The major $\mathrm{Ca}^{2+}$ entry route in most cell types is via the $\mathrm{Ca}^{2+}$ release-activated $\mathrm{Ca}^{2+}$ (CRAC) channels, which are composed of the ORAI channels and the STIMs. The most extensively examined proteins in this regard are the predominant isoforms ORAI1 and STIM1. We found that both STIM1 and ORAI1 were significantly upregulated after FGF2 treatment in MUM2B cells (Figure 2B). Moreover, 2-APB, a well-described SOCE inhibitor, could reverse the increased

A

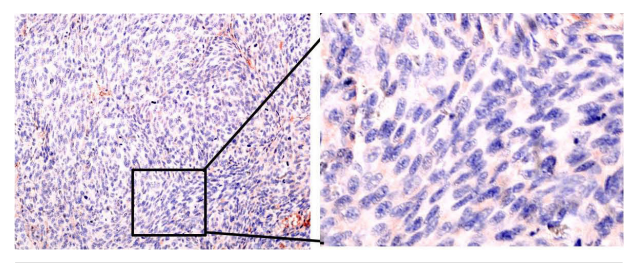

FGF2(-)

B

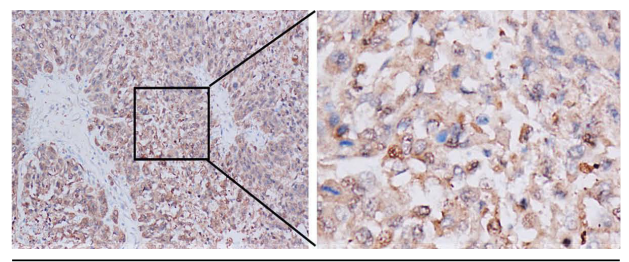

Primary foci

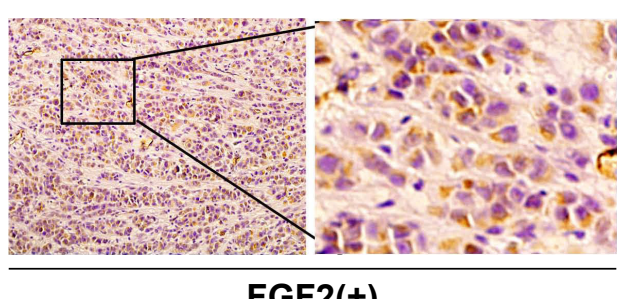

FGF2(+)

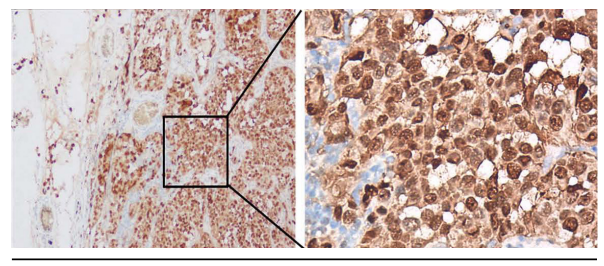

Metastasis foci in lung

Figure I FGF2 expression in human uveal melanoma tissue samples. (A) Representative images of negative FGF2 expression in a spindle cell subtype sample and positive FGF2 expression in an epithelioid subtype sample (immunohistochemical staining, 100x); (B) FGF2 expression was higher in lung metastatic foci than in the primary foci from the same patient (immunohistochemical staining, 100x). 
Table I Correlation between FGF2 and clinicopathologic characteristics of uveal melanoma

\begin{tabular}{|c|c|c|c|}
\hline \multirow[t]{2}{*}{ Variable } & \multicolumn{2}{|c|}{ FGF2 expression (\%) } & \multirow[t]{2}{*}{$P$-value } \\
\hline & - & + & \\
\hline Age (years) & & & 0.211 \\
\hline$<50$ & $2(40.0)$ & $3(60.0)$ & \\
\hline$\geq 50$ & $12(44.4)$ & $15(55.6)$ & \\
\hline Gender & & & 0.928 \\
\hline Male & $8(44.4)$ & $10(55.6)$ & \\
\hline Female & $6(42.9)$ & $8(57.1)$ & \\
\hline LTD (mm) & & & 0.419 \\
\hline$\leq 15$ & $9(50.0)$ & $9(50.0)$ & \\
\hline$>15$ & $5(35.7)$ & $9(64.3)$ & \\
\hline Height (mm) & & & 0.854 \\
\hline$\leq 5$ & $2(40.0)$ & $3(60.0)$ & \\
\hline$>5$ & $12(44.4)$ & $15(55.6)$ & \\
\hline Histological subtype & & & $0.035^{*}$ \\
\hline Spindle cell & $10(83.3)$ & $2(16.7)$ & \\
\hline Mixed/epithelioid & $4(20.0)$ & $16(80.0)$ & \\
\hline Metastasis & & & $0.017^{*}$ \\
\hline Present & $2(16.7)$ & $10(83.3)$ & \\
\hline Absent & $12(60.0)$ & $8(40.0)$ & \\
\hline
\end{tabular}

Note: *Significantly different.

Abbreviation: LTD, largest tumor diameter.

A

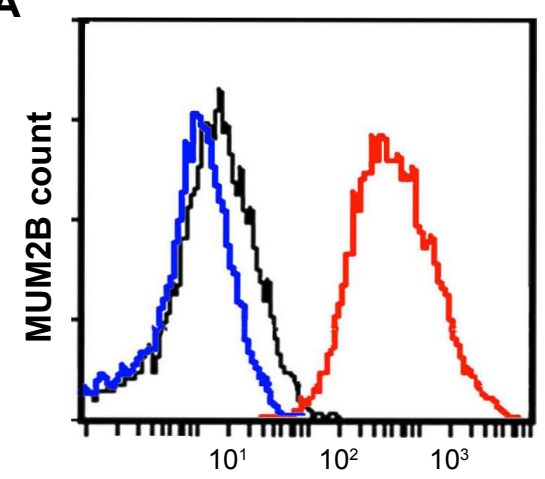

Fluo 3-AM

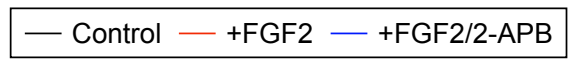

B

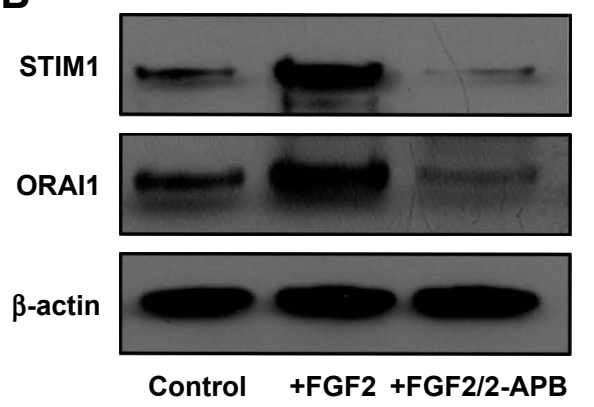

cytosolic $\mathrm{Ca}^{2+}$ concentration and elevated expression of STIM1 and ORAI1 caused by FGF2 (Figure 2), indicating the inhibitory effect of 2-APB on $\mathrm{Ca}^{2+}$ signaling.

\section{2-APB impairs increased migration and decreased adhesion of MUM2B cells induced by FGF2}

To assess the effects of FGF2 on migration in UM cells, we investigated the horizontal and vertical motility abilities of MUM2B UM cells with FGF2 treatment by using the conventional wound healing and Boyden chamber assays. As expected, UM cells with FGF2 stimulation showed significantly increased motile activity, compared with the control cells (Figure 3A and B).

Tumor metastasis is a multistep process, wherein alterations in the adhesive property of neoplastic cells play a pivotal role. Loss of intercellular adhesion and desquamation of cells from the underlying basement membrane allows malignant cells to escape from their site of origin and the surrounding
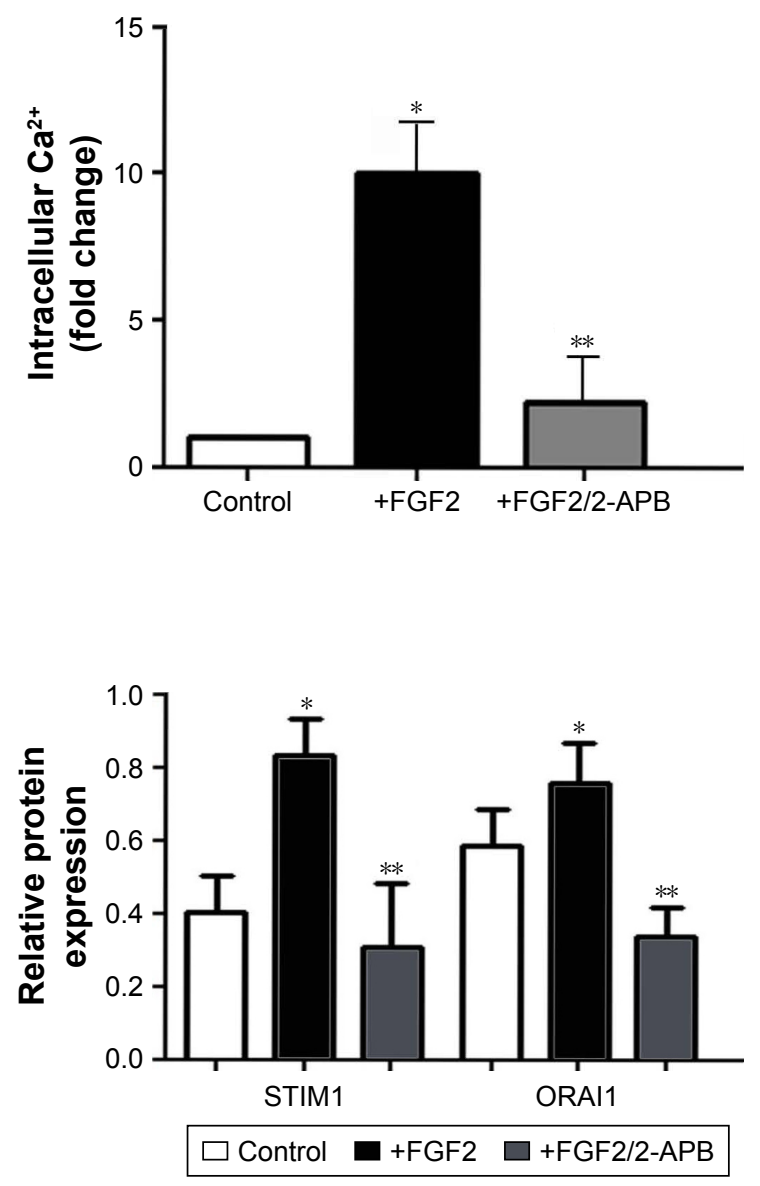

Figure 2 The effect of FGF2 on intracellular $\mathrm{Ca}^{2+}$ concentration and expression of store-operated calcium entry-regulated proteins. (A) FGF2 stimulation elevated intracellular calcium concentration, and 2-APB reversed this effect (left); (B) FGF2 stimulation increased the expression of STIMI and ORAll of MUM2B cells, and 2-APB reversed this effect (left). Relative amounts of protein expression of STIMI and ORAll compared with $\beta$-actin (right) ( $* P<0.05$ between FGF2-treated cells and the control cells, $* * P<0.05$ between FGF2/2-APB-treated cells and the FGF2-treated cells). 
A
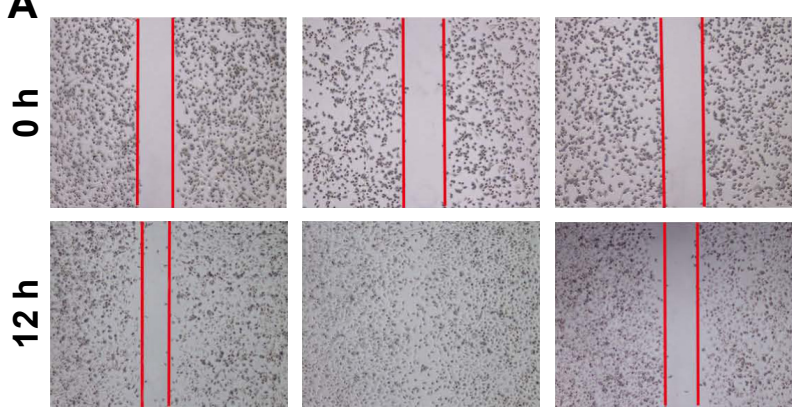

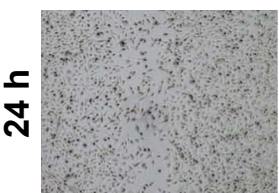

Control

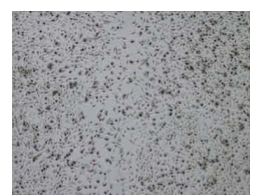

+FGF2

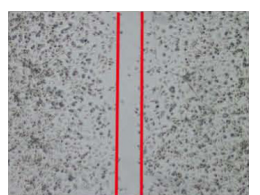

+FGF2/2-APB

B

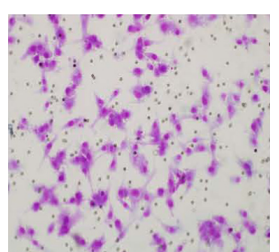

Control

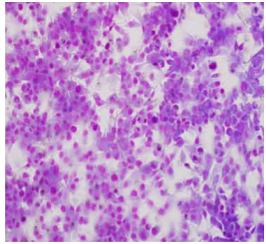

+FGF2

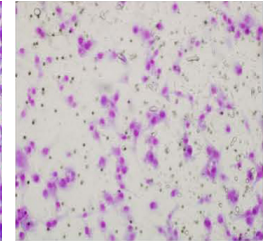

+FGF2/2-APB
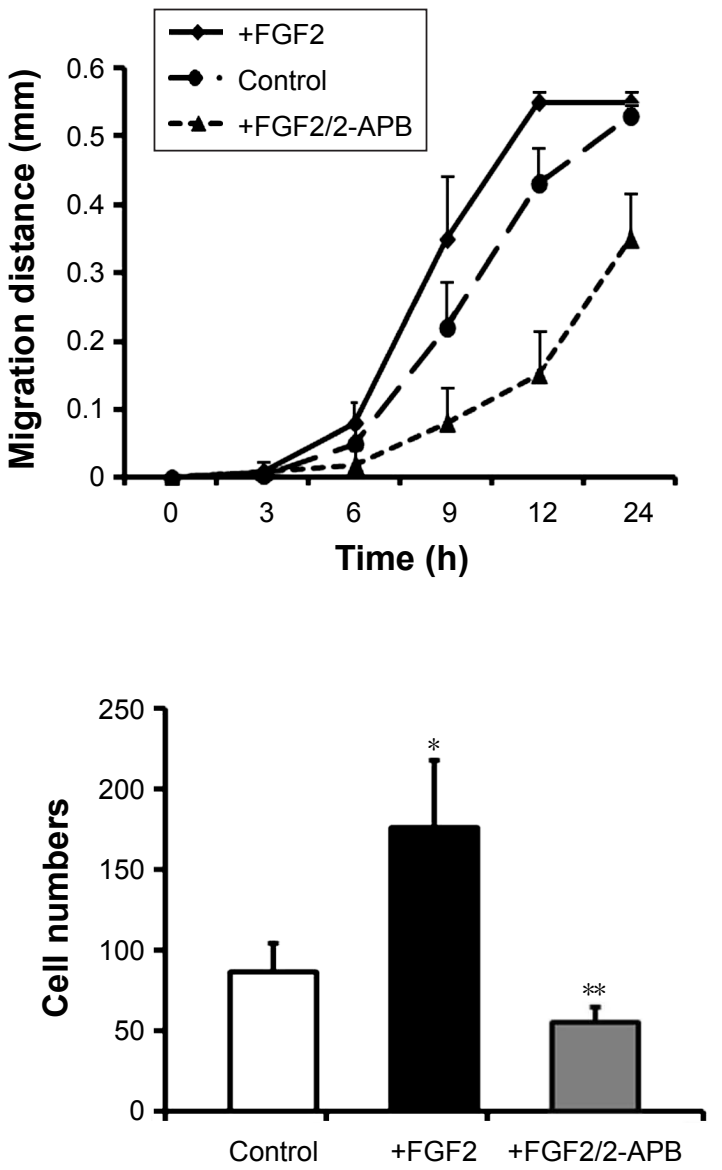

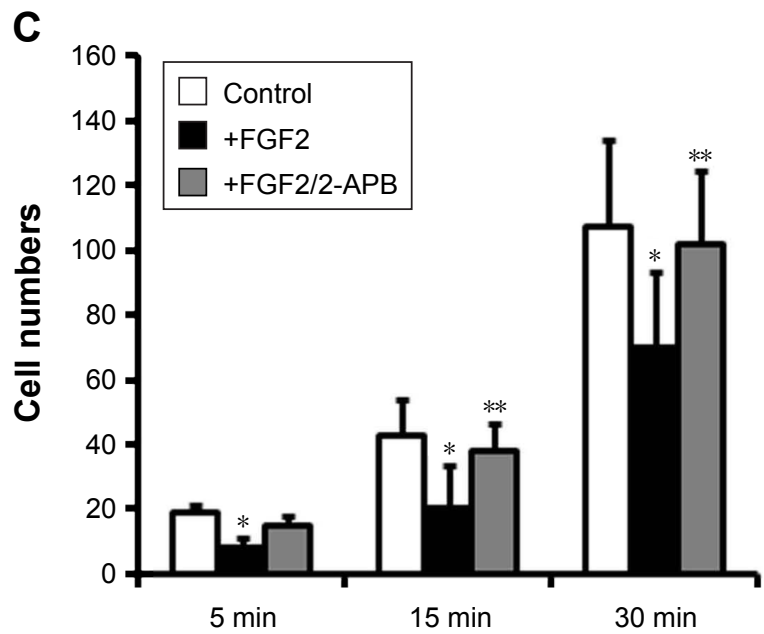

Figure 3 2-APB impairs increased horizontal and vertical migration and decreased adhesion of MUM2B cells caused by FGF2. (A) Images of wound healing assay at 0, I2, and $24 \mathrm{~h}(100 \times)$ (left). Migration distances at different times in the wound healing assay (right); (B) Cells invading through the Boyden chamber were stained (200X) (left). The numbers of invading cells were counted in five predetermined fields (right); (C) The numbers of bound cells were counted in five high magnification $(200 \times)$ fields $(* P<0.05$ between FGF2-treated cells and the control cells, $* * P<0.05$ between FGF2/2-APB-treated cells and the FGF2-treated cells).

barrier, and to acquire a more motile phenotype. So, we also detected the effect of FGF2 on adhesion of UM cells and found that FGF2 caused decreased intercellular adhesion of MUM2B cells (Figure 3C). In the presence of 2-APB, the increased migration of, and the decreased adhesion properties of, MUM2B caused by FGF2 were significantly reversed $(P<0.05$; Figure 3$)$, suggesting that SOCE plays a role in regulating MUM2B cell migration. 


\section{2-APB impairs cytoskeleton assembly of MUM2B cells caused by FGF2}

Cell migration depends on the actin cytoskeleton, which is capable of undergoing rapid cycles of assembly and disassembly. Structural and functional dysregulation of the actin cytoskeleton correlate with uncontrolled movement of tumor cells. By using phalloidin to dye fibrous actin (F-actin), we observed that FGF2 treatment caused cell polarization and pseudopodium protrusion in MUM2B cells (Figure 4). Similarly, 2-APB inhibited cytoskeleton assembly of MUM2B cells with FGF2 treatment, demonstrating SOCE is also involved in FGF2-induced cell migration via altering the actin cytoskeleton.

\section{2-APB inhibits in vivo tumor growth, metastasis caused by FGF2, and decreases the expression of STIMI and ORAII in a MUM2B xenograft mouse model}

Next, we made a mouse xenograft model to assess the effect of FGF2 and 2-APB on tumor growth and metastasis in vivo. MUM2B cells with FGF2 stimulation grew into larger tumor masses, compared with the control cells and cells subjected to FGF2/2-APB treatment $(P<0.05$; Figure 5A). Similar to the in vitro findings, of the 10 mice injected with cells with FGF2 stimulation, one showed lung and liver metastases simultaneously, one showed lung metastasis, and two showed vascular invasion (Figure 5B), whereas no metastatic sites were detected in mice that received FGF2/2-APB treatment.
Immunohistochemical analyses on the sections of xenograft tissues showed that tumor sections from FGF2stimulated cells exhibited a markedly increased expression of STIM1 and ORAI1, whereas sections from control and FGF2/2-APB-stimulated tumor masses revealed no or weak staining. These results substantiate our findings that FGF2 induces SOCE, which contributes to promoting metastasis of UM cells.

\section{FGF2 expression is concomitant with the expression of STIMI and ORAII in human UM tissues}

We then detected the expression of STIM and ORAI1 to analyze the relationship between FGF2 and SOCE in the 32 human UM specimens. As shown in Table 2 and Figure 6, samples with positive FGF2 expression showed higher expression of STIM and ORAI1 $(P<0.05)$ than those with negative FGF2 expression, further indicating the SOCE-promoting effect of FGF2 in UM.

\section{Discussion}

Malignant behaviors in melanoma are stimulated by a variety of growth factors, including FGFs. Expression of FGF2 has been identified as an important characteristic of melanoma cells in contrast to normal melanocytes. ${ }^{22}$ FGF2overexpressing melanocytes exhibit marked proliferation, upward migration, cluster formation, and type IV collagen expression within the epidermal compartment, stimulating early radial growth phase resembling early-stage melanoma. ${ }^{12}$
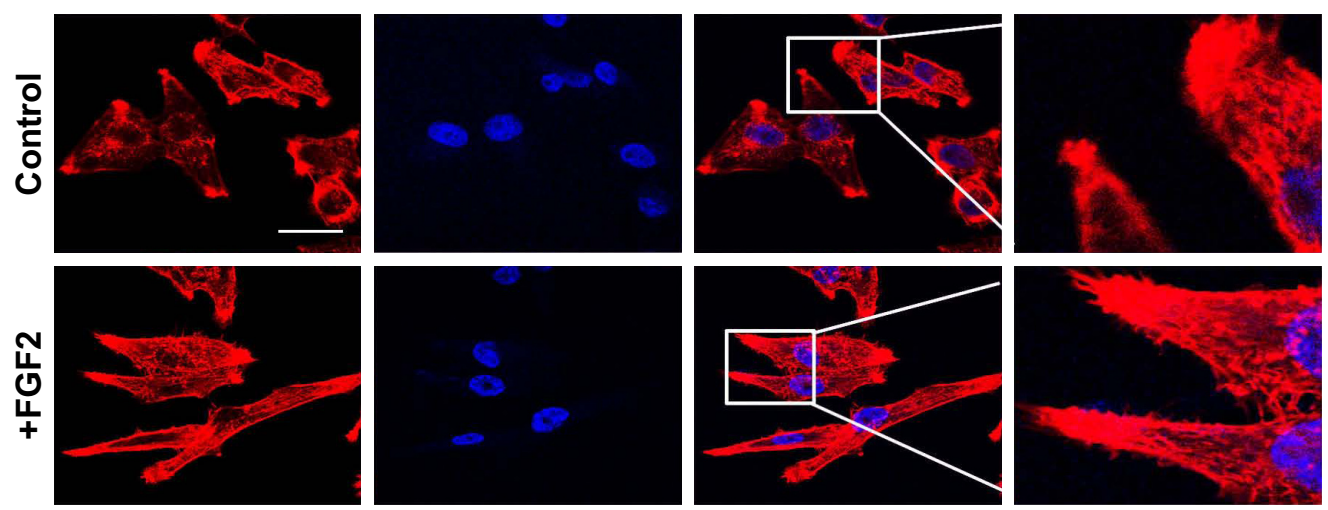

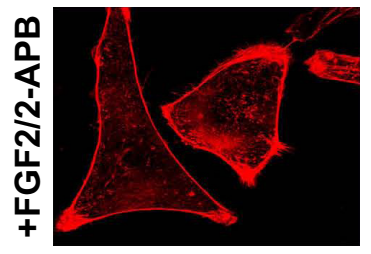

F-actin

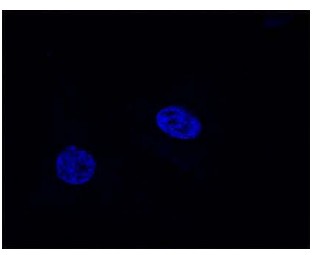

DAPI

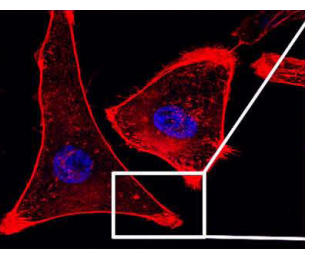

Merge

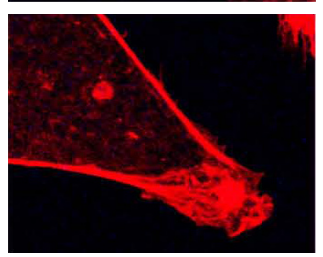

Figure 4 2-APB impairs cytoskeleton assembly of MUM2B cells caused by FGF2. (Immunofluorescent staining of F-actin, Scale bar: $50 \mu \mathrm{m}$ ). Abbreviation: DAPI, diamidino-phenyl-indole. 
A

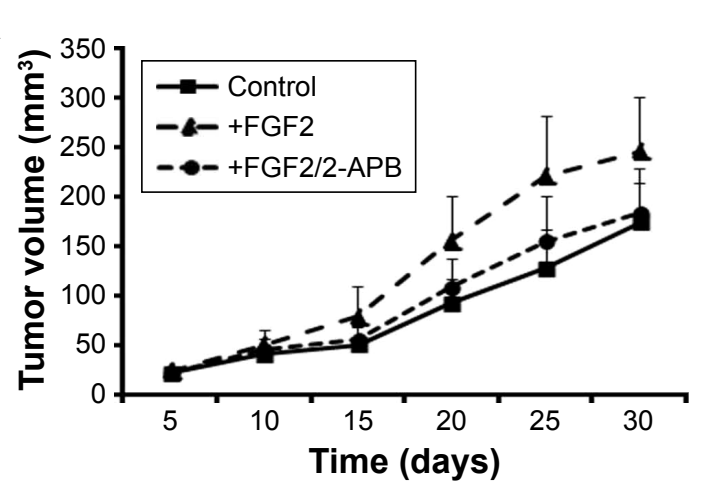

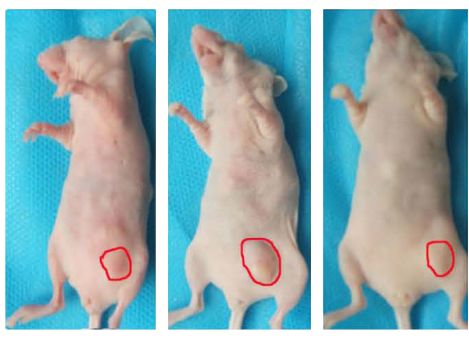

Control

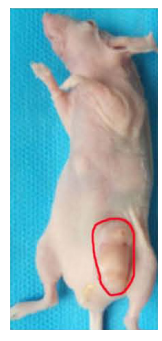

+FGF2

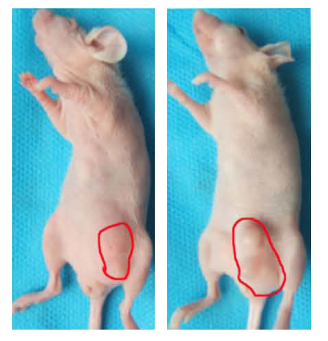

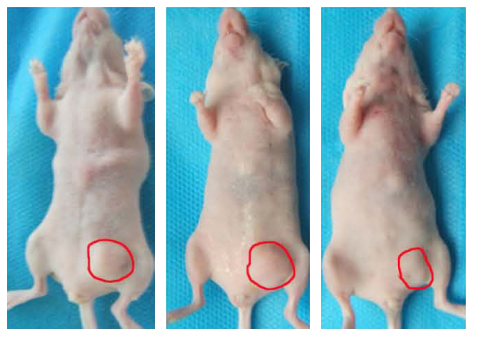

+FGF2/2-APB

B

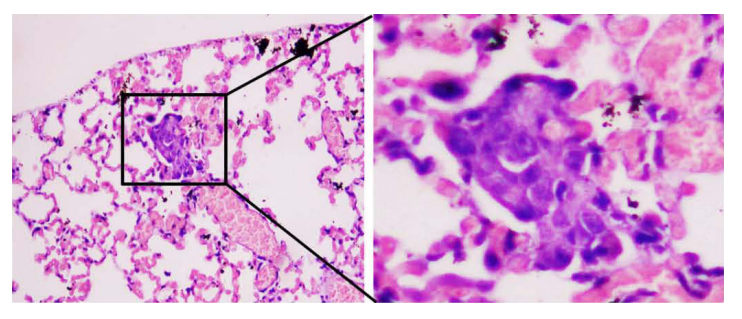

Metastatic colony in lung

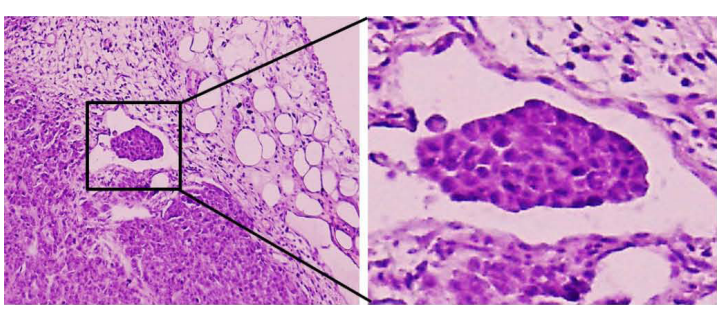

Intravascular tumor thrombus

C
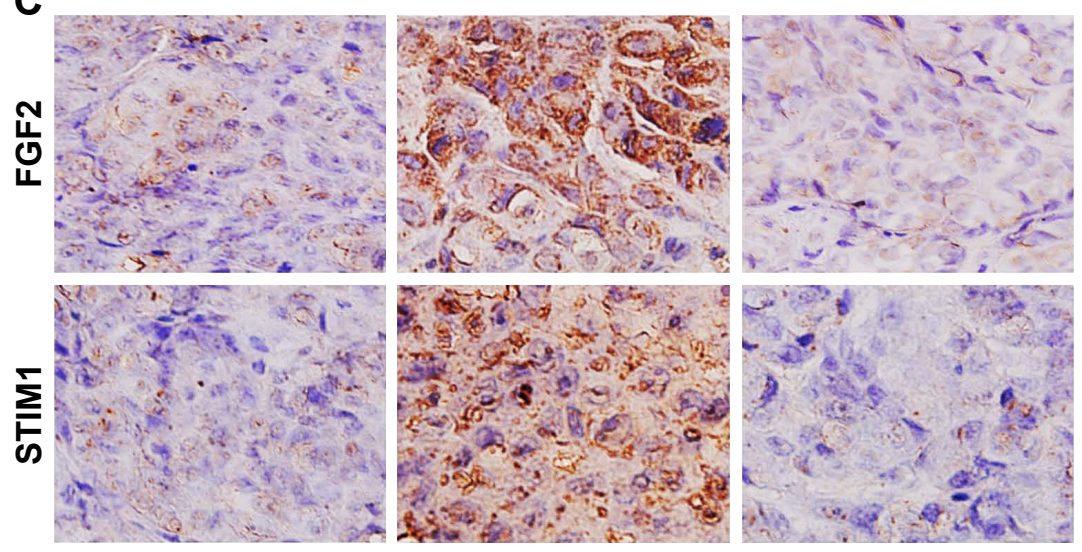

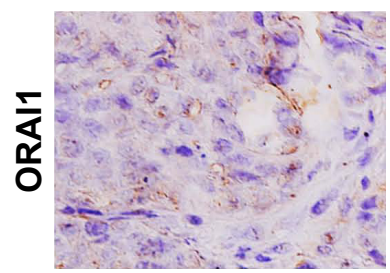

Control

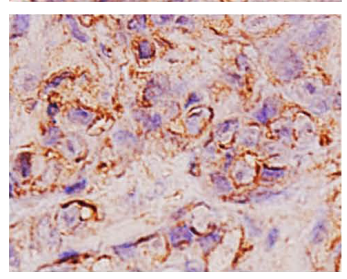

+FGF2

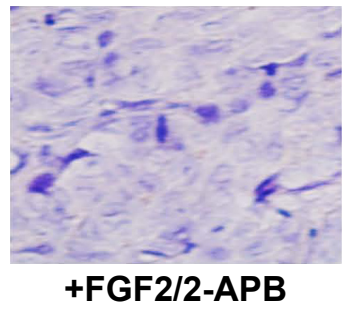

Figure 5 2-APB inhibits in vivo tumor growth, metastasis caused by FGF2 and decreases the expression of STIMI and ORAII in a MUM2B xenograft mouse model. (A) Xenograft tumor volumes are monitored over time (upper). Representative xenograft tumors on Day 30 post injection (lower); (B) Mice injected with cells with FGF2 stimulation showed lung metastasis and vascular invasion (H\&E staining, 200×); (C) Representative images of the expression of FGF2, STIMI, and ORAII in xenograft tumor tissues (immunohistochemical staining, 400x). 
Table 2 Correlation between expression of FGF2 and ORAII, STIMI

\begin{tabular}{|c|c|c|c|c|c|}
\hline \multirow[t]{2}{*}{ Variable } & \multirow[t]{2}{*}{ Total (\%) } & \multicolumn{2}{|c|}{ FGF2 expression } & \multirow[t]{2}{*}{$\chi^{2}$} & \multirow[t]{2}{*}{$P$-value } \\
\hline & & - & + & & \\
\hline \multicolumn{4}{|c|}{ STIMI expression } & 7.158 & $0.0075^{*}$ \\
\hline- & $19(59.4)$ & $12(63.2)$ & $7(36.8)$ & & \\
\hline+ & $13(40.6)$ & $2(15.4)$ & II (84.6) & & \\
\hline \multicolumn{4}{|c|}{ ORAII expression } & 6.026 & $0.014 *$ \\
\hline- & $15(46.9)$ & $10(66.7)$ & $5(33.3)$ & & \\
\hline+ & $17(53.1)$ & $4(23.5)$ & $13(76.5)$ & & \\
\hline
\end{tabular}

Note: *Significantly different.

FGF2 is a mitogen for melanocytes and is produced by the neighboring keratinocytes and fibroblasts. ${ }^{22}$ It has been shown that both FGF2 and FGFR are simultaneously overexpressed in melanoma cells. Thus, FGF2 also functions in an autocrine manner in melanoma. ${ }^{10} \mathrm{FGF} 2$ has been shown to be a potent angiogenic molecule in vivo and in vitro. Coexpression of FGFR-1 and bFGF in melanoma cells was associated with increased microvessel density, and antisense oligonucleotides against FGF2 mRNA could effectively inhibit intratumoral angiogenesis and melanoma growth in nude mice. ${ }^{23,24}$ Lefevre and colleagues reported that FGF2 was strongly expressed in UM, and that antisense oligonucleotide-mediated depletion of endogenous FGF2 could induce decreased proliferation of UM cells. ${ }^{10}$ Although the study was valuable, they undertook Western blotting and polymerase chain reaction in five primary uveal melanoma cell lines and nine UM cell samples from patients, which are less representative owing to the limited sample size. Therefore, we examined FGF2 expression in 32 human UM tissues and showed that the expression of FGF2 was related to the presence of metastasis. Immunohistochemical staining of primary melanomas and metastases revealed a heterogeneous expression of FGF2 in primary and metastatic melanoma, indicating the important role of FGF2 in UM progression. In addition, FGF2 was predominantly present in uveal melanomas of mixed/epithelioid subtype, whereas only two out of 12 spindle cell melanomas showed FGF2 expression, suggesting that FGF2 may also contribute to malignant differentiation of UM.

We observed FGF2 treatment markedly elevated the intracellular calcium concentration and upregulated the expression of STIM1 and ORAI1. In the human UM tissue samples, the FGF2 positive expression group showed higher levels of STIM1 and ORAI1 expression than the negative expression group. Immunostaining of mouse xenograft tissue samples further strengthened the hypothesis that FGF2 is involved in inducing SOCE in UM cells. To our knowledge, this is the first report illustrating the role of FGF2 on SOCE of tumor cells. Activation of FGF2/FGFR signaling generates IP3, which makes $\mathrm{Ca}^{2+}$ release from calcium stores. ${ }^{25}$ SOCE is

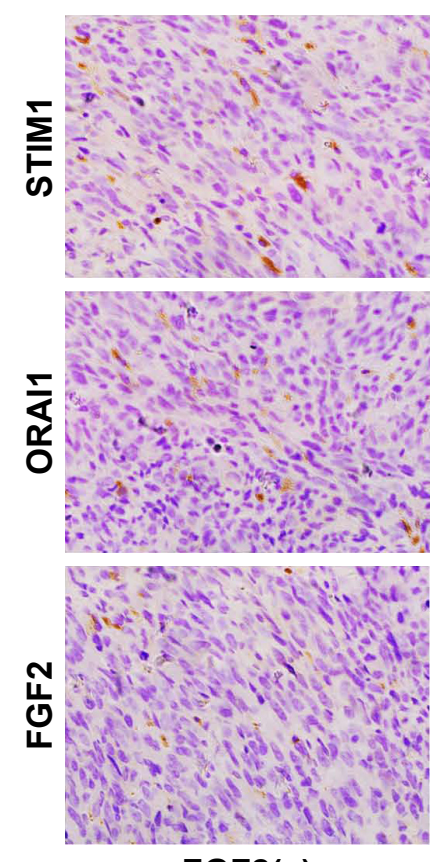

FGF2(-)

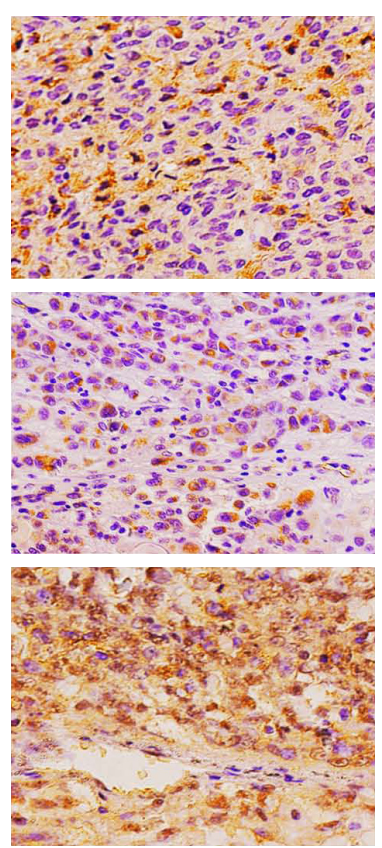

FGF2(+)

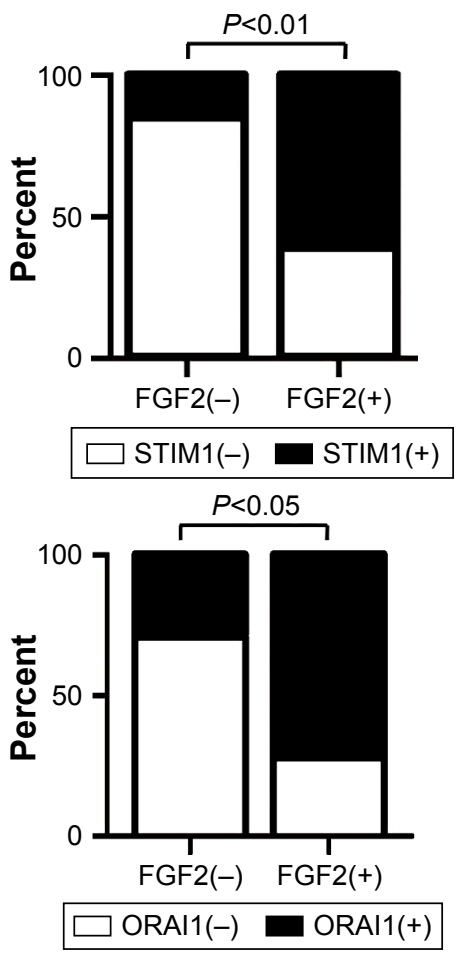

Figure 6 FGF2 expression was concomitant with the expression of STIMI and ORAII in human uveal melanoma tissue samples. Representative images of the expression of FGF2, STIMI, and ORAII in FGF2-negative and -positive groups (immunohistochemical staining, 200x) (left). Percentages of STIMI or ORAII negative and positive expression in the FGF2-negative and FGF2-positive groups (right). 
a major mechanism of $\mathrm{Ca}^{2+}$ import from the extracellular to intracellular space following depletion of $\mathrm{Ca}^{2+}$ stores in non-excitable cells. ${ }^{26}$ STIM1 and ORAI1, two important determinants of $\mathrm{Ca}^{2+}$ homeostasis and SOCE, have been reported to be upregulated in most cancers, suggesting a resultant increase in SOCE. ${ }^{27-30}$ Feldman et al showed that ORAI1 and STIM1 expressed in B16BL6 mouse melanoma cells control SOCE, and silencing of STIM1 caused a reduction in cell growth and increased cell death. ${ }^{31}$ Another study in melanoma, published by Sun et al, showed that ORAI and STIM are involved in invadopodia formation and tumor invasion. ${ }^{32}$ Several other studies showed that ORAI and STIM-mediated SOCE correlated with the upregulation of proteins, such as microphthalmia-associated transcription factor (MITF), that indicate a proliferative melanoma cell phenotype, and the downregulation of slow-cycling cell markers such as JARID1B and Brn2. ${ }^{33-35}$

As a ubiquitous secondary messenger, intracellular calcium regulates the normal function and pathologies associated with a wide variety of cells. Localized elevations in intracellular $\mathrm{Ca}^{2+}$ interplay with filamin A, $\alpha$-actinin, gelsolin, villin, scinderin, severin, or calcineurin B to initiate assembly of the invadopodium, responsible for appropriate traction. ${ }^{36-38}$ Moreover, calcium signaling regulates the activation and translocation of Rac, which is implicated in tumor progression. ${ }^{39}$ There has been accumulating evidence suggesting that hyperactive SOCE endows tumor cells with special properties related to their metastatic phenotypes. ${ }^{16}$ ORAI1 blockade was reported to inhibit the recycling of MT1-matrix metalloproteinase (MMP) and extracellular matrix degradation. ${ }^{32}$ Umemura et al reported that STIM1 knockdown significantly suppressed melanoma lung metastasis in a xenograft mouse model, implicating the importance of SOCE in metastatic dissemination. ${ }^{40} \mathrm{We}$ found FGF2 treatment increased horizontal and vertical migration and $\mathrm{F}$-actin cytoskeleton assembly, decreased adhesive activity of MUM2B UM cells, together with promotion of metastasis in a mouse xenograft model. The SOCE pharmacological inhibitor 2-APB could abrogate the pro-migration response to FGF2 in vitro, and restrain tumor metastasis in the mouse xenograft model. Thus, FGF2-induced tumor metastasis correlates with enhancement of SOCE, and the antimotility effect caused by 2-APB in UM may be caused by its inhibitory effect on SOCE. Moreover, our study lends further support for the possible benefit of 2-APB or other SOCE inhibitors as adjuvant therapies for FGF2-dependent tumors, including UM.

Multiple signaling pathways involved in tumor growth and progression were observed downstream of FGF2, such as the MAPK, PI3K/AKT, PLC, and STAT pathways. ${ }^{25}$ One study in melanoma showed that induction of SOCE was associated with activation of extracellular-signal-regulated kinase (ERK). ${ }^{40}$ Schmidt et al reported that ORAI1/STIM1 expression was increased in therapy-resistant ovarian carcinoma cells, which at least in part is due to enhanced Akt activity. ${ }^{41}$ Studies designed to elucidate the interactions of SOCE with other FGF2-associated signaling pathways in UM are currently underway. In addition, we have to point out the existence of discrepant findings. Some researchers found disruption of the FGF2 gene could not affect tumor growth in an experimental model of hemangiomas, nor prevent tumor growth and angiogenesis in a transgenic mouse model for tumors of the retinal pigment epithelium. ${ }^{42,43}$ Stanisz et al reported that silencing of ORAI1/STIM2 caused a decrease in intracellular $\mathrm{Ca}^{2+}$, which correlated with increased expression of microphthalmiaassociated transcription factor and enhanced proliferation. ${ }^{44}$ These discrepancies indicate a clear need for additional studies examining the effect and mechanisms of FGF2 and $\mathrm{Ca}^{2+}$-related processes in the progression of different types of tumor.

\section{Conclusion}

FGF2 promotes metastasis of UM cells and this property may at least in part be due to enhanced SOCE. The results might have a number of implications for establishment of clinically useful therapeutic targets aimed at UM in the future.

\section{Acknowledgment}

This study was supported by the National Natural Science Foundation of China (grant no 81360145). The use of the tissue samples in this study was approved by the Institutional Research Committee of the Second Affiliated Hospital of Chongqing Medical University. All patients provided written informed consent for the use of their tissue samples for future research. The Institutional Animal Use and Care Committee of Chongqing Medical University approved the in vivo experiments, and the animal care and handling procedures followed their guidelines (or similar, for example, the Guide for the Care and Use of Laboratory Animals is a widely acknowledged and accepted set of rules).

\section{Author contributions}

All authors made substantial contributions to conception and design, acquisition of data, or analysis and interpretation of data; took part in drafting the article or revising it critically for important intellectual content; gave final approval of the version to be published; and agree to be accountable for all aspects of the work.

\section{Disclosure}

The authors report no conflicts of interest in this work. 


\section{References}

1. Shields CL, Shields JA. Ocular melanoma: relatively rare but requiring respect. Clin Dermatol. 2009;27(1):122-133.

2. Singh AD, Turell ME, Topham AK. Uveal melanoma: trends in incidence, treatment, and survival. Ophthalmology. 2011;118(9):1881-1885.

3. Augsburger JJ, Corrêa ZM, Shaikh AH. Effectiveness of treatments for metastatic uveal melanoma. Am J Ophthalmol. 2009;148(1):119-127.

4. Song X, Dai D, He X, et al. Growth factor FGF2 cooperates with interleukin-17 to repair intestinal epithelial damage. Immunity. 2015; 43(3):488-501.

5. Zhang Y, Wang S, Wang X, Liao S, Wu Y, Han C. Endogenously produced FGF2 is essential for the survival and proliferation of cultured mouse spermatogonial stem cells. Cell Res. 2012;22(4):773-776.

6. Price CA. Mechanisms of fibroblast growth factor signaling in the ovarian follicle. J Endocrinol. 2016;228(2):R31-R43.

7. Slattery ML, John EM, Stern MC, et al. Associations with growth factor genes (FGF1, FGF2, PDGFB, FGFR2, NRG2, EGF, ERBB2) with breast cancer risk and survival: the Breast Cancer Health Disparities Study. Breast Cancer Res Treat. 2013;140(3):587-601.

8. Suzuki T, Yasuda H, Funaishi K, et al. Multiple roles of extracellular fibroblast growth factors in lung cancer cells. Int J Oncol. 2015;46(1): 423-429.

9. Li Z, Sasaki Y, Mezawa M, et al. cAMP and fibroblast growth factor 2 regulate bone sialoprotein gene expression in human prostate cancer cells. Gene. 2011;471(1-2):1-12.

10. Lefèvre G, Babchia N, Calipel A, et al. Activation of the FGF2/FGFR1 autocrine loop for cell proliferation and survival in uveal melanoma cells. Invest Ophthalmol Vis Sci. 2009;50(3):1047-1057.

11. Ozen M, Medrano EE, Ittmann M. Inhibition of proliferation and survival of melanoma cells by adenoviral-mediated expression of dominant negative fibroblast growth factor receptor. Melanoma Res. 2004;14(1):13-21.

12. Meier F, Caroli U, Satyamoorthy K, et al. Fibroblast growth factor-2 but not Mel-CAM and/or beta3 integrin promotes progression of melanocytes to melanoma. Exp Dermatol. 2003;12(3):296-306.

13. Ronca R, Di Salle E, Giacomini A, et al. Long pentraxin-3 inhibits epithelial-mesenchymal transition in melanoma cells. Mol Cancer Ther. 2013;12(12):2760-2771.

14. Brewer JR, Mazot P, Soriano P. Genetic insights into the mechanisms of Fgf signaling. Genes Dev. 2016;30(7):751-771.

15. Prakriya M, Lewis RS. Store-operated calcium channels. Physiol Rev. 2015;95(4):1383-1436.

16. Xie J, Pan H, Yao J, Zhou Y, Han W. SOCE and cancer: recent progress and new perspectives. Int J Cancer. 2016;138(9):2067-2077.

17. Chen YF, Chiu WT, Chen YT, et al. Calcium store sensor stromalinteraction molecule 1-dependent signaling plays an important role in cervical cancer growth, migration, and angiogenesis. Proc Natl Acad Sci US A. 2011;108(37):15225-15230.

18. Qi L, Song W, Li L, et al. FGF4 induces epithelial-mesenchymal transition by inducing store-operated calcium entry in lung adenocarcinoma. Oncotarget. 2016;7(45):74015-74030.

19. Wong JR, Nanji AA, Galor A, Karp CL. Management of conjunctival malignant melanoma: a review and update. Expert Rev Ophthalmol. 2014;9(3):185-204.

20. Folberg R, Kadkol SS, Frenkel S, et al. Authenticating cell lines in ophthalmic research laboratories. Invest Ophthalmol Vis Sci. 2008; 49(11):4697-4701.

21. Folberg R, Arbieva Z, Moses J, et al. Tumor cell plasticity in uveal melanoma: microenvironment directed dampening of the invasive and metastatic genotype and phenotype accompanies the generation of vasculogenic mimicry patterns. Am J Pathol. 2006;169(4):1376-1389.

22. Halaban R, Langdon R, Birchall N, et al. Basic fibroblast growth factor from human keratinocytes is a natural mitogen for melanocytes. $J$ Cell Biol. 1988;107(4):1611-1619.
23. Li D, Wang H, Xiang JJ, et al. Monoclonal antibodies targeting basic fibroblast growth factor inhibit the growth of B16 melanoma in vivo and in vitro. Oncol Rep. 2010;24(2):457-463.

24. Xiao L, Yang S, Hao J, et al. Endostar attenuates melanoma tumor growth via its interruption of b-FGF mediated angiogenesis. Cancer Lett. 2015;359(1):148-154.

25. Ornitz DM, Marie PJ. Fibroblast growth factor signaling in skeletal development and disease. Genes Dev. 2015;29(14):1463-1486.

26. Redondo PC, Rosado JA. Store-operated calcium entry: unveiling the calcium handling signalplex. Int Rev Cell Mol Biol. 2015;316:183-226.

27. Xia J, Wang H, Huang H, et al. Elevated Orail and STIM1 expressions upregulate MACC1 expression to promote tumor cell proliferation, metabolism, migration, and invasion in human gastric cancer. Cancer Lett. 2016;381(1):31-40.

28. Tang BD, Xia X, Lv XF, et al. Inhibition of Orai1-mediated Ca2+ entry enhances chemosensitivity of HepG2 hepatocarcinoma cells to 5-fluorouracil. J Cell Mol Med. 2017;21(5):904-915.

29. Vashisht A, Trebak M, Motiani RK. STIM and Orai proteins as novel targets for cancer therapy. A Review in the Theme: cell and molecular processes in cancer metastasis. Am J Physiol Cell Physiol. 2015; 309(7):C457-C469.

30. Li Y, Guo B, Xie Q, et al. STIM1 mediates hypoxia-driven hepatocarcinogenesis via interaction with HIF-1. Cell Rep. 2015;12(3):388-395.

31. Feldman B, Fedida-Metula S, Nita J, Sekler I, Fishman D. Coupling of mitochondria to store-operated $\mathrm{Ca}(2+)$-signaling sustains constitutive activation of protein kinase B/Akt and augments survival of malignant melanoma cells. Cell Calcium. 2010;47(6):525-537.

32. Sun J, Lu F, He H, et al. STIM1- and Orail-mediated $\mathrm{Ca}(2+)$ oscillation orchestrates invadopodium formation and melanoma invasion. $J$ Cell Biol. 2014;207(4):535-548.

33. Hoek KS, Goding CR. Cancer stem cells versus phenotype-switching in melanoma. Pigment Cell Melanoma Res. 2010;23(6):746-759.

34. Roesch A, Vultur A, Bogeski I, et al. Overcoming intrinsic multidrug resistance in melanoma by blocking the mitochondrial respiratory chain of slow-cycling JARID1B(high) cells. Cancer Cell. 2013;23(6): 811-825.

35. Stanisz H, Stark A, Kilch T, et al. ORAI1 Ca(2+) channels control endothelin-1-induced mitogenesis and melanogenesis in primary human melanocytes. J Invest Dermatol. 2012;132(5):1443-1451.

36. Stossel TP, Fenteany G, Hartwig JH. Cell surface actin remodeling. J Cell Sci. 2006;119(Pt 16):3261-3264.

37. Robinson RC, Mejillano M, Le VP, Burtnick LD, Yin HL, Choe S. Domain movement in gelsolin: a calcium-activated switch. Science. 1999;286(5446):1939-1942.

38. Nakamura F, Hartwig JH, Stossel TP, Szymanski PT. Ca2+ and calmodulin regulate the binding of filamin A to actin filaments. J Biol Chem. 2005;280(37):32426-32433.

39. Price LS, Langeslag M, ten Klooster JP, Hordijk PL, Jalink K, Collard JG. Calcium signaling regulates translocation and activation of Rac. J Biol Chem. 2003;278(41):39413-39421.

40. Umemura M, Baljinnyam E, Feske S, et al. Store-operated Ca2+ entry (SOCE) regulates melanoma proliferation and cell migration. PLoS One. 2014;9(2):e89292.

41. Schmidt S, Liu G, Liu G, et al. Enhanced Orail and STIM1 expression as well as store operated $\mathrm{Ca} 2+$ entry in therapy resistant ovary carcinoma cells. Oncotarget. 2014;5(13):4799-4810.

42. Tobe T, Ortega S, Luna JD, et al. Targeted disruption of the FGF2 gene does not prevent choroidal neovascularization in a murine model. Am J Pathol. 1998;153(5):1641-1646.

43. Foletti A, Ackermann J, Schmidt A, Hummler E, Beermann F. Absence of fibroblast growth factor 2 does not prevent tumor formation originating from the RPE. Oncogene. 2002;21(12):1841-1847.

44. Stanisz H, Saul S, Müller CS, et al. Inverse regulation of melanoma growth and migration by Orai1/STIM2-dependent calcium entry. Pigment Cell Melanoma Res. 2014;27(3):442-453. 


\section{Publish your work in this journal}

OncoTargets and Therapy is an international, peer-reviewed, open access journal focusing on the pathological basis of all cancers, potential targets for therapy and treatment protocols employed to improve the management of cancer patients. The journal also focuses on the impact of management programs and new therapeutic agents and protocols on

patient perspectives such as quality of life, adherence and satisfaction. The manuscript management system is completely online and includes a very quick and fair peer-review system, which is all easy to use. Visit http://www.dovepress.com/testimonials.php to read real quotes from published authors.

Submit your manuscript here: http://www.dovepress.com/oncotargets-and-therapy-journal 